\title{
Strategies and Methods in the Identification of Antagonists of Protein-Protein Interactions
}

\author{
Thomas R. Gadek \\ Genentech, South San Francisco, CA, USA \\ BioTechniques 34:S21-S24 (June 2003)
}

\begin{abstract}
The identification of antagonists of protein-protein interactions is a critical challenge to the pharmaceutical industry. The selection of a protein target, which is amenable to antagonism, is the first of many decisions that determine the success of these efforts. In addition, the definition of strategies and the development and application of methodologies appropriate to that target will be vital to the success of efforts to identify antagonists of a protein-protein interaction. An analysis of current approaches to the identification of lead molecules demonstrates that a search for competitors of a known binder is the basis of traditional screening as well as more modern approaches based on structure activity relationship (SAR) by nuclear magnetic resonance (NMR), molecular fragments, rational design, and tethering. The latter methods employ a structural perspective, throughout the discovery and optimization of a lead, to provide the practitioner with some control over the success of the process.
\end{abstract}

\section{INTRODUCTION}

Interactions between proteins, whether enzyme-substrate, receptor-ligand, or other protein-protein interactions, are critical to the spatial, temporal, and functional distribution of proteins within cells, tissues, and organisms. These processes underlie the biology of human disease, and the control of these interactions could be critical to the treatment of those disease states. The discovery of small molecule leads in the inhibition of large proteinprotein interactions has been recognized as one of the major research challenges facing the pharmaceutical industry in the $21 \mathrm{st}$ century (1). A series of recent reviews have covered aspects of these efforts and noted successes utilizing screening, rational design, structure activity relationship (SAR) by nuclear magnetic resonance (NMR), NMR-guided fragment selection, and phage display in the search for these leads (2-7). These methods are largely extensions of existing lead discovery and optimization technologies as applied to this challenging problem. Additional methods and strategies have been developed specifically to facilitate the discovery of small molecule antagonists of large protein-protein interactions (8-11). When applied to the identification and optimization of antagonists of protein-protein interactions, these newer structurally guided approaches seek to provide the practitioner with a perspective or common point of reference throughout the process. This can provide a sense of direction to the hunt as well as to the hunter(s). The noted reviews (2-7) have been thorough in their coverage of these topics and approaches, and the reader is referred to them for the details. This review will attempt to provide the reader with perspective on aspects of these approaches, which have enabled their successful application, and some discussion of the scope, limitations, and challenges facing of the field.

\section{PROTEIN INTERACTIONS}

An examination of protein surfaces in direct contact with each other have indicated that the topography of these contacts is relatively flat in comparison to the deep crevices, pockets, and holes that have dominated the analysis of protease active sites and the binding of substrates and/or inhibitors. This difference in perception has fostered thoughts of the difficulty of the challenge in inhibiting a protein-protein interaction. However, it should be noted that these interacting protein surfaces are not featureless, and they present each other with complementary electronic and topographical contours that are involved in the initial binding attraction and subsequent stabilization of the interaction between the proteins of interest $(8,12,13)$. Consequently, a suitable small molecule, which binds to the surface of a target protein and forms a stable complex with the protein by presenting a complementary surface, can block the binding of the cognate protein ligand by either a competitive or allosteric mechanism (14). In the case of the competitive inhibitor, the protein-small molecule complex will project a modified electronic and topographical surface, which is no longer attractive or complementary to its native protein partner. The allosteric antagonist will form a complex with the target protein, which has a modified topography and/or diminished affinity for the epitope of its partner.

In this simplistic view, small molecule antagonists of large protein-protein interactions should be relatively easy to find, but this has not been the experience of researchers in the field. 
In fact, there is a common belief among medicinal chemists that these inhibitors are impossible or at least very difficult to find. The reality of the challenge lies somewhere between these extreme views. Key to the successful identification of lead structures has been the very selection of the target protein within the protein-protein interaction of interest. This protein should have a surface-binding site that is critical to the binding of the protein partner. As noted above, antagonism of the protein-protein interaction by a small molecule occupying this site can be competitive or noncompetitive in nature. The competitive inhibitors can bind to a site that overlaps with the contact surface of the native ligand, while the noncompetitive inhibitors may bind to a non-overlapping site that controls the affinity of the target protein for it's cognate protein ligand (e.g., an allosteric inhibition). In either case, the small molecule antagonist must be able to provide a complementary electrostatic (i.e., polar and nonpolar) surface contour to the target protein, which provides binding energy to the complex sufficient to effectively compete with the binding of the native protein ligand. In order to be considered a practical lead to a pharmaceutical, this must be achieved within a small molecule framework of $<500$ molecular weight and a solvent-accessible surface area of 150-500 $\AA^{2}$ $(6,13,15,16)$. While the typical protein-protein interaction involves a contact surface area of $1600 \AA^{2}$ (13), an effective small molecule need not occupy this entire space. An antagonist of this size would not be expected to have reasonable pharmaceutical properties and would not be considered a small molecule lead $(15,16)$. Consequently, it seems that it is the size and affinity of the small molecule, not the actual size of the interacting proteins, that are the keys to the successful search for a small molecule inhibitor of a large protein-protein interaction (6). Generally, a realistic combination of these properties in a molecule, which could be considered a lead toward a pharmaceutical, would be high affinity (e.g., $\mathrm{kDa}<10 \mathrm{nM}$ ) and a binding footprint on the surface of the target protein that is equal to or less than that of a steroid.

\section{LEAD IDENTIFICATION}

\section{Screening}

Antagonists of a protein-protein interaction have been identified using screening assays that label one of the protein partners in a search for a molecule that blocks the association of the proteins. This strategy can utilize a number of assay formats (e.g., ELISA, radiolabel, fluorescence, enzyme activity, in silico screening) to identify molecules that bind to either of the proteins in the interaction and is capable of identifying competitive and noncompetitive antagonists (2). The primary assay used for these purposes can employ the purified proteins or can involve cell culture where either the fate or phenotype of the cells reflect the functional inhibition of a protein-protein interaction. The initial successes in the identification of small molecule antagonists of a protein interaction preceded the advent of molecular biology and our ability to clone and express the proteins involved in the interaction and relied on activities observed in animals or cell culture to identify a lead. Once a small molecule antagonist is identified, a labeled form of it can be used in a sub- sequent competition assay to identify additional antagonists that compete with the initial small molecule lead for a common binding site on the target protein. This is a proven approach frequently employed in either a back-up or "fast follower" mode within pharmaceutical research organizations.

The identification of inhibitors of tubulin polymerization exemplify these aspects of screening in the identification of small molecule leads (17). The vinca alkaloids were isolated in the mid-1950s from the Madigascar periwinkle, Catharanthus roseus, and were noted to suppress bone marrow and cause granulocytopenia in rats (18). Once this pharmacological activity was noted in animals, it was used to guide the isolation of vincristine and vinblastine and to elucidate their mechanism of action. These lead compounds were subsequently tested for their ability to treat acute lymphocytic neoplasm and have been a frontline therapy in the treatment of human cancers for more than 40 years $(17,19)$. The use of labeled forms of these leads greatly facilitated the discovery of numerous additional natural products, which are also active against tumors (20). It was only in the mid-1990s that these compounds were shown to bind to $\beta$-tubulin, thus blocking the formation of the $\alpha \beta$-tubulin heterodimer and the assembly of the mitotic spindle (21). Ultimately, these efforts have enabled the rational design of newer classes of agents that bind to the same vinblastine site on $\beta$ tubulin (22-24). Numerous additional examples of lead molecules that have been identified by screening are discussed in several recent reviews $(2,5)$. It should be noted that these more modern lead discovery efforts take advantage of advances in the field since the discovery of the vinca alkaloids. In general, these efforts use purified recombinant proteins in a competitive binding assay or cell culture experiments to find inhibitors of a protein-protein interaction.

\section{Phage Display}

Phage display mutagenesis is a combinatorial screening technology that links the phenotype and genotype of a virus in a process that selects for those phage that bind to the target of interest $(7,25,26)$. Typically, small peptide sequences are inserted into one of the coat proteins in a manner that will allow their display on the outer surface of the phage's coat protein. These sequences can be varied in a combinatorial sense to display a large library of potential binders that can be readily selected, amplified, and sequenced to identify high affinity binders. This methodology has been used to study the energetics of proteinprotein interfaces to define the SAR of the interacting proteins (27), as well as to identify small peptide ligands of a protein of interest (7). This technique has proven successful against nearly every protein it has been targeted to and has been suggested to be the method of choice in testing the feasibility of finding an inhibitor of a given protein-protein interaction. The format can be customized to select for peptide sequences that are selective for the target protein and either competitive (28) or noncompetitive (29) in their inhibition of a protein-protein interaction. These peptide sequences bind to the target protein when displayed on the surface of phage and as synthetic short linear and cyclic peptides independent of the phage. In some instances, these peptides have been leads in the identification of nonpeptides $(7,16,28,29)$. 


\section{NMR Techniques}

NMR has been used to detect small molecules that interact with numerous target proteins. When applied to the inhibition of protein-protein interactions, this technique has had success in the identification and optimization of small molecule antagonists. In particular, the recent discovery of immunosuppressive lymphocyte function-associated antigen-1 (LFA-1) antagonists has exemplified the power of NMR-based drug discovery strategies. Utilizing the I domain, a 200-amino acid fragment of the $\alpha$-subunit of LFA-1, workers at Abbott Laboratories (Abbott Park, IL, USA) were able to develop an SAR for this protein, the protein in complex with antagonists, and of the antagonists themselves $(4,30,31)$. These studies enabled the development and execution of a novel strategy of lead optimization based on the optimization of fragments or substructures. In this approach, a lead molecule known to bind to the target protein is deconstructed into a set of fragments, alternative substructures are identified, which compete with each fragment, and novel series of compounds with improved affinity and pharmaceutical properties are identified in a subsequent (re)assembly of these alternative fragments. In the case of LFA-1, this provided compounds with improved potency, solubility, half-life, and oral bioavailability (31). This fragment approach, when coupled with the SARs developed for the protein and the small molecules, allowed the medicinal chemists to solve the LFA- 1 antagonist puzzle by feeling their way along the surface of LFA-1, testing the fit of the pieces of the puzzle (i.e., fragments) for the correct electronic and contour complementarity required of an antagonist of a protein-protein interaction.

\section{Rational Discovery}

The proteins involved in a protein-protein interaction have themselves been used as a source of small molecule leads $(6,32,33)$. The study of protein structure, coupled with studies of the effect of individual amino acid side chains on the binding and/or function of the protein, provide an SAR for the protein, which defines its epitope (34). When this epitope is key to a protein-protein interaction, mimicry of the SAR of a cognate protein ligand can provide a point of departure for the rational discovery of potent antagonists $(6,35)$. This approach has been exemplified in the discovery of a class of intercellular adhesion molecule type-1 (ICAM-1) mimetics as antagonsists of the LFA-1/ICAM-1 interaction (36). Alanine point mutagenesis was used to identify the side chain functionality of six residues comprising a contiguous nonlinear binding epitope within the first immunoglobulin domain of ICAM-1. A homology model was constructed to define the SAR of ICAM-1, and aspects of this SAR were recast in several series of low affinity peptide analogs of ICAM-1 (37). A computational correlation of the affinity and conformational dynamics of these peptides defined a likely bound conformation, which was the basis of a rational transfer of ICAM-1's epitope to a small molecule framework. The optimization of these small molecule leads followed a fragment strategy and resulted in the identification of a compound 20 -fold more potent than cyclosporine in the inhibition of lymphocyte proliferation (36). Again, an appreciation of protein structure and function, in comparison with antagonist structure and function, provided the perspective enabling the rapid identification and optimization of an antagonist of a protein-protein interaction. It is interesting to note the similar strategies and successes achieved in the identification of LFA-1 antagonists were based on this example of the mimicry of the ICAM epitope and the earlier example, which complemented the LFA-1 epitope. The former identified a positive image/active analog of a known binder (i.e., ICAM-1), while the latter constructed a negative image complementary to the electronic and topographic contours of LFA-1.

\section{Tethering}

Recently, a novel approach to the problem of identifying antagonists of proteins has emerged, which captures and covalently tethers a complementary fragment molecule to the surface of a target protein at a defined location (10). This fragment can be combined with other similarly identified fragments to produce a molecule with enhanced affinity that combines the binding contacts of each fragment. Ultimately, the functional group used to tether the fragment(s) can be eliminated from the combined fragments to produce a high affinity lead molecule. This method has been applied to identify antagonists of interleukin-2 (IL-2) and its interaction with IL-2 receptor $(8,9)$. Surface residues surrounding the binding site of a known antagonist (38) were modified to incorporate a reactive thiol group of a cysteine in a series of mutants of IL-2 (9). A library of fragment molecules containing a disulfide moiety was screened against the panel cysteine mutants under partial reducing conditions, which promotes rapid exchange between thiols and disulfides. Members of the disulfide-containing library with an inherent affinity for IL2 at a site in proximity to the free thiol are captured, identified, and merged into the structure of the known antagonist. A structural characterization of this process revealed that binding of the small molecule antagonist to the surface of IL-2 is an adaptive process with significant rearrangement of the protein surface in the formation of the protein-small molecule complex (9). Utilizing this approach and aspects of the fragment strategy discussed earlier, an analog of a known IL-2 antagonist was enhanced in affinity 50 -fold (8).

\section{CONCLUSION}

Just as proteins adapt in forming a protein-protein interaction or a complex with a small molecule antagonist, researchers pursuing antagonists must adapt strategies and methodologies in response to knowledge of the structure and function of the target protein. Along this line, a common theme in the successful examples discussed in this review is the emerging use of the fragment approach to antagonist optimization. This allows an active series of small molecule analogs to adapt to the target protein surface in the course of their optimization. In addition, a structural perspective on the protein target or its interaction partner can provide a template upon which hypothesis concerning the SAR of small molecule antagonists can be formulated, tested, and refined. Similarly, considerations of the SAR of the protein target itself can identify those proteins involved in a protein-protein interaction that are amenable to inhibition. Ad- 


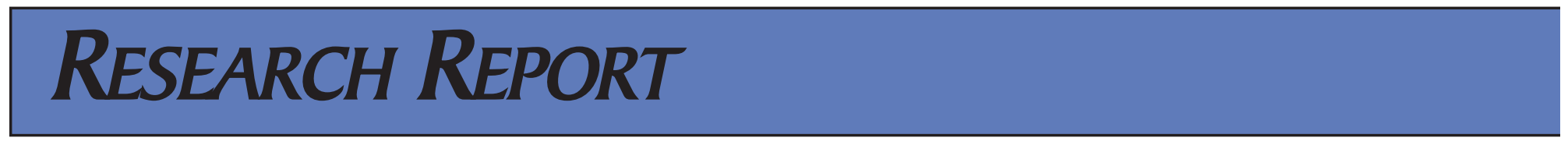

vances in structural biology, spectroscopic, and computational methodology over the last 10 years have enabled a more detailed perspective on the structural and functional commonalities between protein-protein interactions and their antagonists. These common threads seem to be guiding research efforts that have successfully inhibited these interactions.

\section{REFERENCES}

1.Hirschmann, R.F. 2003. Foreward. In C. Wermuth (Ed.), The Practice of Medicinal Chemistry, 2nd ed. Elsevier, St. Louis.

2.Toogood, P.L. 2002. Inhibition of protein-protein association by small molecules: approaches and progress. J. Med. Chem. 45:1543-1558.

3.Fesik, S.W., S.B. Shuker, P.J. Hajduk, and R.P.Meadows. 1997. SAR by NMR: an NMR-based approach for drug discovery. Protein Eng. 10(Suppl):73.

4.Huth, J.R. and C. Sun. 2002. Utility of NMR in lead optimization: fragment-based approaches. Comb. Chem. High Throughput Screen. 5:631643.

5.Ockey, D.A. and T.R. Gadek. 2002. Inhibitors of protein-protein interactions. Expert Opin. Ther. Pat. 12:393-400.

6.Gadek, T.R. and J.B. Nicholas. 2003. Small molecule antagonists of proteins. Biochem. Pharmacol. 65:1-8.

7.Sidhu, S.S., W.J. Fairbrother, and K. Deshayes. 2003. Exploring proteinprotein interactions with phage display. Chem. Biochem. 4:14-25.

8.Braisted, A.C., J.D. Oslob, W.L. Delano, J. Hyde, R.S. McDowell, N. Waal, C. Yu, M.R. Arkin, and B.C. Raimundo. 2003. Discovery of a potent small molecule IL-2 inhibitor through fragment assembly. J. Am. Chem. Soc. 125:3714-3715.

9.Arkin, M.R., M. Randal, W.L. DeLano, J. Hyde, T.N. Luong, J.D. Oslob, D.R. Raphael, L.Taylor, et al. 2003. Binding of small molecules to an adaptive protein-protein interface. Proc. Natl. Acad. Sci. USA 100:1603-1608.

10.Erlanson, D.A., A.C. Braisted, D.R. Raphael, M. Randal, R.M. Stroud, E.M. Gordon, and J.A. Wells. 2000. Site-directed ligand discovery. Proc. Natl. Acad. Sci. USA 97:9367-9372.

11.Erlanson, D.A., J.W. Lam, C. Wiesmann, T.N. Luong, R.L. Simmons, W.L. DeLano, I.C. Choong, M.T. Burdett, et al. 2003. In situ assembly of enzyme inhibitors using extended tethering. Nat. Biotechnol. 21:308-314.

12.Janin, J. 1995. Principles of protein-protein recognition from structure to thermodynamics. Biochemie 7:497-505.

13.Lo Conte, L., C. Chothia, and J. Janin. 1999. The atomic structure of protein-protein recognition sites. J. Mol. Biol. 285:2177-2198.

14.Pratt, W.B. and P. Taylor. 1990. Principles of Drug Action: The Basis of Pharmacology. Churchill Livingston, New York.

15.Lipinski, C.A., F. Lomnbardo, B.W. Dominy, and P.J. Feeney. 1997. Experimental and computatiuonal approaches to estimate solubility and permeability in drug discovery and development settings. Adv. Drug Deliv. Rev. 23:3-25.

16. Rishton, G.M. 2003. Nonleadlikeness and leadlikeness in biochemical screening. Drug Discov. Today 8:86-96.

17.Calibrisi, P. and B.A. Chabner. 2001. p.1417-1419. In J.G. Hardman and L.E. Limbird (Eds.), Goodman and Gilman's The Pharmacological Basis of Therapeutics. McGraw-Hill, New York.

18.Noble, R.L., C.T. Beer, and J.H. Cutts. 1958. Role of chance observations in chemotherapy: Vinca rosea. Ann. NY Acad. Sci. 76:882-894.

19. Warwicke, O.H., J.M. Darte, T.C. Brown, C.T. Beer, J.H. Cutts, and R.L. Noble. 1960. Some biological effects of vincaleukoblastine, an alkaloid in Vinca rosea Linn in patients with malignant disease. Cancer Res. 20:103221040.

20.Hamel, E. 1992. Natural products which interact with tubulin in the vinca domain: maytansine, rhizoxin, phomopsinA, dolastatins 10 and 15 and halichondrin B. Pharmacol. Ther. 55:331-51.

21.Rai, S.S. and J. Wolff. 1996. Localization of the vinblasine-binding site on beta tubulin. J. Biol. Chem. 271:14707-14711.

22.Jordan, A., J.A. Hadfield, N.J. Lawrence, and A.T. McGown. 1998. Tubulin as a target for anticancer drugs: agents which interact with the mitotic spindle. Med. Res. Rev. 18:259-296.

23.Von Angerer, E. 1999. New inhibitors of tubulin polymerization. Expert Opin. Ther. Pat. 9:1069-1081.
24.Uckun, F.M., E.A. Sudbeck, C. Mao, S. Ghosh, X.-P. Liu, A.O. Vassilev, C.S. Navara, and R.K. Narla. 2001. Structure-based design of novel anticancer agents. Curr. Cancer Drug Targets 1:59-71.

25.Smith, G.P. 1985. Filamentous fusion phage: novel expression vectors that display cloned antigens on the virion surface. Science 14:1315-1317.

26.Bass, S., R. Greene, and J.A. Wells. 1990. Hormone phage: an enrichment method for variant proteins with altered binding properties. Proteins 8:309314.

27.Weiss, G.A., C.K. Watanabe, A. Zhong, A. Goddard, and S.S. Sidhu. 2000. Rapid mapping of protein functional epitopes by combinatorial alanine scanning. Proc. Natl. Acad. Sci. USA 97:8950-8954.

28.Dubree, N.J.P., D.R. Artis, G. Castanedo, J. Marsters, D. Sutherlin, L. Caris, K. Clark, S.M. Keating, et al. 2002. Selective $\alpha 4 \beta 7$ integrin antagonists and their potential as anti-inflammatory agents. J. Med. Chem. 45:3451-3457.

29.Dennis, M.S., C. Eigenbrot, N.J. Skelton, M.H. Ultsch, L. Santell, M.A. Dwyer, M.P. O’Connell, and R.A. Lazarus. 2000. Peptide exosite inhibitors of factor VIIa as anticoagulants. Nature 404:465-470.

30.Huth, J.R., E.T. Olejniczak, R. Mendoza, H. Liang, E.A.S. Harris, M.L. Lupher, Jr., A.E. Wilson, S.W. Fesik, and D.E. Staunton. 2000. NMR and mutagenesis evidence for an I domain allosteric site that regulates lymphocyte function-associated antigen 1 ligand binding. Proc. Natl. Acad. Sci. USA 97:5231-5236.

31.Lui, G., J.R. Huth, E.T. Olejniczak, R. Mendoza, P. DeVries, S. Leitza, E.B. Reilly, G.F. Okasinski, et al. 2001. Novel p-arylthio cinnamides as antagonists of leukocyte function-associated antigen-1/intracellular adhesion molecule-1 interaction. 2. Mechanism of inhibition and structure-based improvement of pharmaceutical properties. J. Med. Chem. 44:1202-1210.

32.Freidinger, R.M. 1999. Nonpeptidic ligands for peptide and protein receptors. Curr. Opin. Chem. Biol. 3:395-406.

33.Ripka, A.S. and D.H. Rich. 1998. Peptidomimetic design. Curr. Opin. Chem. Biol. 2:441-452.

34.Cunningham, B.C. and J.A. Wells. 1993. Comparison of a structural and a functional epitope. J. Mol. Biol. 234:554-563.

35.de Vos, A.M., M.H. Ultsch, A.A. Kossiakoff, G. Fuh, B.C. Cunningham, and J.A. Wells. 1995. Rational design of potent antagonists to the human growth hormone receptor. Hum. Growth Horm. Pharmacol. 295:29-41.

36.Gadek, T.R., D.J. Burdick, R.S. McDowell, M.S. Stanley, J.C. Marsters, Jr., K.J. Paris, D.A. Oare, M.E. Reynolds, et al. 2002. Generation of an LFA-1 antagonist by the transfer of the ICAM-1 immunoregulatory epitope to a small molecule. Science 295:1086-1089.

37.Fisher, K.L., J. Lu, L. Riddle, K.J. Kim, L.G. Presta, and S.C. Bodary. 1997. Identification of the binding site in intercellular adhesion molecule1 for its receptor, leukocyte function-associated antigen 1. Mol. Biol. Cell. 8:501-515.

38.Tilley, J.W., L. Chen, D.C. Fry, S.D. Emerson, G.D. Powers, D. Biondi, T. Varnell, R. Trilles, et al. 1997. Identification of a small molecule inhibitor of the IL-2/IL-2R receptor interaction which binds to IL-2. J. Am. Chem. Soc. 119:7589-7590.

\section{Address correspondence to:}

Thomas R. Gadek

Medicinal Chemistry Department

Genentech, Inc.

One DNA Way

South San Francisco, CA 94080, USA

e-mail:trg@gene.com 